\title{
On divisors whose sum is a square
}

\author{
by \\ WOLFGANG JENKNER (Wien)
}

1. Introduction. We are interested in the arithmetic function

$$
a(n)=\#\left\{(x, y) \in \mathbb{N} \times \mathbb{N}_{0} \mid x^{4}-y^{2}=4 n,(x, y)=1\right\} .
$$

It is related to the family of elliptic curves $E_{n}: \eta^{2}=\xi^{3}+n \xi(n \in \mathbb{N})$ by means of the birational transformation $E_{n} \rightarrow \bar{E}_{n}$ given by

$$
\left\{\begin{array}{l}
\bar{\xi}=\frac{\eta}{\xi}, \\
\bar{\eta}=\frac{\eta^{2}-2 \xi^{3}}{\xi^{2}}
\end{array}\right.
$$

with $\bar{E}_{n}: \bar{\xi}^{4}-\bar{\eta}^{2}=4 n$ (cf. [4], 64.X, $\S 6$ ). We assume $\bar{\xi}>0$, so that we can write

$$
\bar{\xi}=\frac{\bar{x}}{\bar{z}}, \quad \bar{\eta}=\frac{\bar{y}}{\bar{z}}
$$

with $\bar{x}, \bar{y}, \bar{z} \in \mathbb{Z}, \bar{x}>0, \bar{z}>0$ and $(\bar{x}, \bar{y}, \bar{z})=1$. It is easy to see that there are $x, z \in \mathbb{N}$ with $(x, z)=1$ so that

$$
\bar{z}=z^{2}, \quad \bar{x}=x z .
$$

So we have to deal with the equation

$$
x^{4}-y^{2}=4 n z^{4} \quad \text { with }(x, z)=1 .
$$

Note that for $x, y, z$ satisfying this equation, the condition $(x, z)=1$ is equivalent to $\left(\left(x^{2}-y\right) / 2,\left(x^{2}+y\right) / 2, z\right)=1$, which implies

$$
\left(x^{2}-y\right) / 2=p^{4} d, \quad\left(x^{2}+y\right) / 2=q^{4} t
$$

with $p q=z,(p, q)=1, d t=n$ and $p^{4} d+q^{4} t=x^{2}$ for some positive integers $p, q, d, t$.

In fact, this is just a special case of a classical method for determining the rank of certain elliptic curves over $\mathbb{Q}$ (see [2]); in particular, for square-free $n, a(n)$ and the rank $r_{n}$ of $E_{n}$ are related by the inequality $2^{r_{n}+1} \geq a(n)$.

1991 Mathematics Subject Classification: 11D25, 11G05, 11N25. 
Some aspects of the closely related arithmetic function counting all lattice points (not just the primitive ones) $(x, y)$ with $x^{4}-y^{2}=4 n$ are described in [1]. An asymptotic expansion for its arithmetic mean is a special case of the results in [3].

In the following section we will consider the slightly more general case of the function

$$
a_{\lambda}(n)=\#\left\{(x, y) \in \mathbb{N} \times \mathbb{N}_{0} \mid \lambda^{2} x^{4}-y^{2}=4 n,(x, y)=1\right\}
$$

for some fixed $\lambda \in \mathbb{N}$.

2. The arithmetic mean. Our goal in this section is to establish the following result.

Proposition 1. Let $T \geq 1$. Then

$$
\sum_{n \leq T} a_{\lambda}(n)=C \frac{(4 T)^{3 / 4}}{\lambda^{1 / 2}}+O\left(T^{1 / 2} \log (T / \lambda+e)\right)
$$

with

$$
C=\frac{1}{3} \cdot \frac{1}{\zeta(2)} \cdot \frac{1}{6} \cdot \frac{\Gamma(1 / 4) \Gamma(1 / 2)}{\Gamma(3 / 4)}=\frac{\Gamma(1 / 4)^{2}}{3 \sqrt{2} \pi^{5 / 2}} .
$$

P r o of. In order not to encumber the notation, we write out the proof only for $\lambda=1$. Setting $S=4 T$, we may express the sum as

$$
\sum_{x \leq \sqrt{S}} \#\left\{y \in \mathbb{N}_{0} \mid x^{4}-S \leq y^{2}<x^{4}, x \equiv y \bmod 2,(x, y)=1\right\} .
$$

As usual, we can dispense with the last condition by means of the Möbius function, which gives

$$
\sum_{n} \mu(n) \sum_{x \leq \sqrt{S} / n} \#\left\{y \in \mathbb{N}_{0} \mid n^{2} x^{4}-S / n^{2} \leq y^{2}<n^{2} x^{4}, x n \equiv y n \bmod 2\right\} .
$$

In order to eliminate the annoying congruence, we observe that for the principal character $\chi \bmod 2$ and $a, b \in \mathbb{N}_{0}$,

$$
(1-\chi(a))(1-\chi(b))+\chi(a) \chi(b)= \begin{cases}1 & \text { if } a \equiv b \bmod 2, \\ 0 & \text { otherwise. }\end{cases}
$$

In view of this relation, we find it convenient to consider sums

$$
\sum_{n} \mu(n) \chi_{1}(n) \sum_{x \leq \sqrt{S} / n} \chi_{2}(x) \sum_{n^{2} x^{4}-S / n^{2} \leq y^{2}<n^{2} x^{4}} \chi_{3}(y),
$$

where $\chi_{i}(i=1,2,3)$ are the principal characters $\bmod N_{i} \in \mathbb{N}$ (however, with a view to applying (3), we need only $\left.N_{i} \in\{1,2\}\right)$. 
Splitting the last sum, we get

$$
\begin{aligned}
& \sum_{n \leq S^{1 / 4}} \mu(n) \chi_{1}(n) \sum_{x \leq S^{1 / 4} / n} \chi_{2}(x) \sum_{y<n x^{2}} \chi_{3}(y) \\
& \quad+\sum_{n \leq S^{1 / 2}} \mu(n) \chi_{1}(n) \sum_{S^{1 / 4} / n<x \leq S^{1 / 2} / n} \chi_{2}(x) \sum_{\sqrt{n^{2} x^{4}-S / n^{2}} \leq y<n x^{2}} \chi_{3}(y),
\end{aligned}
$$

which gives after a routine calculation involving Euler's summation formula and some trivial estimations

$$
I \frac{\phi\left(N_{2}\right) \phi\left(N_{3}\right)}{\zeta(2) N_{2} N_{3} \prod_{p \mid N_{1}}\left(1-1 / p^{2}\right)} S^{3 / 4}+O\left(S^{1 / 2} \log S\right),
$$

where

$$
I=\frac{1}{3}+\int_{1}^{\infty}\left(t^{2}-\sqrt{t^{4}-1}\right) d t=\frac{1}{6} \cdot \frac{\Gamma(1 / 4) \Gamma(1 / 2)}{\Gamma(3 / 4)}
$$

3. The quadratic mean. From now on, we restrict our attention to the case $\lambda=1$.

Proposition 2. Let $T \geq 2$. Then

$$
\sum_{n \leq T} a(n)(a(n)-1) \ll T^{1 / 2}(\log T)^{5} .
$$

P r o of. In view of what was said in the introduction, we have to count the quadruples $\left(d_{1}, t_{1}, d_{2}, t_{2}\right)$ with $\left\{d_{1}, t_{1}\right\} \neq\left\{d_{2}, t_{2}\right\}$ such that $d_{1}+t_{1}$ and $d_{2}+t_{2}$ are squares and $d_{1} t_{1}=d_{2} t_{2}$. The last condition is equivalent to

$$
\frac{d_{1}}{t_{2}}=\frac{d_{2}}{t_{1}}=\frac{a}{b}
$$

for some relatively prime $a$ and $b$, which means that there exist $s$ and $t$ such that

$$
\begin{cases}d_{1}=s a, & d_{2}=t a \\ t_{2}=s b, & t_{1}=t b .\end{cases}
$$

As a result, we have to count the quadruples $(a, b, s, t)$ with

$$
\left\{\begin{array}{l}
a b s t \leq T, \\
s a+t b=\square, \quad t a+s b=\square, \\
a \neq b, \quad s \neq t .
\end{array}\right.
$$

Note that if such a quadruple satisfies these conditions the same holds for $(b, a, s, t),(a, b, t, s)$ and $(s, t, a, b)$, which implies in particular that we can assume $a>b, s>t$ and $a b \leq s t$. 
Let $\nu \in \mathbb{N}$ be a square. First, we count the sextuples $(a, b, s, t, x, y)$ of natural numbers satisfying

$$
\left\{\begin{array}{l}
s t a b \leq T \\
s a+t b=\nu x^{2}, \\
s b+t a=\nu y^{2}, \\
(s a, t b)=(s b, t a)=1, \\
s>t, \quad a>b, \\
a b \leq \sqrt{T}, \quad x \neq y .
\end{array}\right.
$$

Obviously, $(\nu, s t a b)=1$ and the two linear equations in $s$ and $t$ of (4) show that $\nu \mid\left(a^{2}-b^{2}\right)$. Putting $m=\left(a^{2}-b^{2}\right) / \nu$ and actually solving these equations, we get

$$
s=\frac{a x^{2}-b y^{2}}{m}, \quad t=\frac{a y^{2}-b x^{2}}{m} .
$$

So the problem of counting the sextuples satisfying (4) is reduced to finding all solutions $(a, b, x, y)$ of the following system of congruences:

$$
\left\{\begin{array}{c}
a^{2} \equiv b^{2} \bmod \nu \\
a x^{2} \equiv b y^{2} \bmod m, \\
a y^{2} \equiv b x^{2} \bmod m .
\end{array}\right.
$$

Let $a$ and $b$ be fixed. In view of $(a, b)=1$, the definition of $m$ implies

$$
(a, m)=(b, m)=1,
$$

and so (6) shows

$$
(x, m)=(y, m)=1,
$$

and, in fact, the last two congruences of (6) are equivalent. Thus, we are left with the problem of counting solutions $(\varrho \bmod m, x, y)$ satisfying congruences $\bmod m$

$$
\left\{\begin{array}{l}
\varrho^{2} \equiv b / a \\
y \equiv \varrho x
\end{array}\right.
$$

The number of solutions of the first of these congruences equals 0 or the number of residue classes $\tau \bmod m$ with

$$
\tau^{2} \equiv 1 \bmod m \text {. }
$$

Writing this as the equivalent system of congruences modulo powers of the various prime numbers dividing $m$, we find that this number is $\ll 2^{\omega(m)}$, where $\omega(m)$ denotes the number of different primes dividing $m$. Now, for each $\varrho \bmod m$ we have to count all possible $(x, y)$. We begin with the simple observation that for all $T>0, B>A>0$ the number of such pairs satisfying

$$
(x, y)=1, \quad 0<x \leq T, \quad A \leq y / x \leq B
$$


is at most $1+(B-A) T^{2}$. Namely, let $K$ be this number and suppose $K>1$. Dividing the interval $[A, B]$ in the $K-1$ successive intervals of length $(B-A) /(K-1)$, we find two pairs $(x, y)$ and $\left(x^{\prime}, y^{\prime}\right)$ such that

$$
0<\frac{y}{x}-\frac{y^{\prime}}{x^{\prime}} \leq \frac{B-A}{K-1} .
$$

But then this difference

$$
\frac{y x^{\prime}-y^{\prime} x}{x x^{\prime}}
$$

actually equals at least $T^{-2}$, which proves the assertion. Now, assuming that $\varrho$ is a positive member of its residue class, we can write

$$
y=\varrho x-z m
$$

with $z>0$ since $y<x$. Further, $(x, y)=1$ implies $(z, x)=1$. Remembering (5), we see that the condition abst $\leq T$ is equivalent to

$$
f\left(\frac{y^{2}}{x^{2}}\right) \leq \frac{4 T}{x^{4} \nu^{2}},
$$

where we have set

$$
f(t)=\frac{4 a b}{\left(a^{2}-b^{2}\right)^{2}}(a-b t)(a t-b) .
$$

This function is increasing in $[b / a, 1]$ and $f(1)=4 a b /(a+b)^{2}$.

We have to consider two cases.

First case:

$$
\frac{4 T}{x^{4} \nu^{2}} \geq \frac{4 a b}{(a+b)^{2}} \quad \text { or } \quad x \leq\left(\frac{T}{a b}\right)^{1 / 4}\left(\frac{a+b}{\nu}\right)^{1 / 2} .
$$

In this case, we have to count the relatively prime $(x, y)$ such that

$$
\left(\frac{b}{a}\right)^{1 / 2}<\frac{y}{x} \leq 1
$$

which means

$$
(\varrho-1) \frac{\nu}{a^{2}-b^{2}} \leq \frac{z}{x}<\left(\varrho-\left(\frac{b}{a}\right)^{1 / 2}\right) \frac{\nu}{a^{2}-b^{2}} .
$$

The preceding considerations show that this number is at most

$$
\begin{aligned}
\left(\frac{T}{a b}\right)^{1 / 2}\left(\frac{a+b}{\nu}\right)\left(1-\left(\frac{b}{a}\right)^{1 / 2}\right) \frac{\nu}{a^{2}-b^{2}}+1 & \\
& =\frac{T^{1 / 2}}{a b^{1 / 2}\left(a^{1 / 2}+b^{1 / 2}\right)}+1 \leq \frac{T^{1 / 2}}{a^{3 / 2} b^{1 / 2}}+1 .
\end{aligned}
$$


Second case:

$$
\frac{4 T}{x^{4} \nu^{2}}<\frac{4 a b}{(a+b)^{2}} \quad \text { or } \quad x>\left(\frac{T}{a b}\right)^{1 / 4}\left(\frac{a+b}{\nu}\right)^{1 / 2} .
$$

We have to count the $(x, y)$ such that

$$
\left(\frac{b}{a}\right)^{1 / 2}<\frac{y}{x} \leq t^{1 / 2}
$$

where $t$ is the smaller solution of the quadratic equation

$$
f(t)=\frac{4 T}{x^{4} \nu^{2}},
$$

which means

$$
\begin{aligned}
0 & <\frac{y}{x}-\left(\frac{b}{a}\right)^{1 / 2} \\
& <\left(\frac{a^{2}+b^{2}-\left(a^{2}-b^{2}\right)\left(1-4 T x^{-4} \nu^{-2}\right)^{1 / 2}}{2 a b}\right)^{1 / 2}-\left(\frac{b}{a}\right)^{1 / 2},
\end{aligned}
$$

this expression being

$$
\begin{aligned}
& <\frac{\frac{a^{2}+b^{2}-\left(a^{2}-b^{2}\right)\left(1-4 T x^{-4} \nu^{-2}\right)^{1 / 2}}{2 a b}-\frac{b}{a}}{2\left(\frac{b}{a}\right)^{1 / 2}} \\
& =\frac{\left(a^{2}-b^{2}\right)}{4 a^{1 / 2} b^{3 / 2}}\left(1-\left(1-4 T x^{-4} \nu^{-2}\right)^{1 / 2}\right) \leq \frac{\left(a^{2}-b^{2}\right)}{4 a^{1 / 2} b^{3 / 2}} \cdot \frac{4 T}{x^{4} \nu^{2}} .
\end{aligned}
$$

Substituting (7), we find

$$
0<\left(\varrho-\frac{b^{1 / 2}}{a^{1 / 2}}\right) \frac{\nu}{a^{2}-b^{2}}-\frac{z}{x}<\frac{1}{a^{1 / 2} b^{3 / 2}} \cdot \frac{T}{x^{4} \nu},
$$

and so there are at most

$$
\frac{1}{a^{1 / 2} b^{3 / 2}} \cdot \frac{4 T}{u^{2} \nu}+1
$$

suitable $(x, z)$ with $u \leq x \leq 2 u$ for some

$$
u \geq u_{0}:=\left(\frac{T}{a b}\right)^{1 / 4}\left(\frac{a+b}{\nu}\right)^{1 / 2} .
$$

Now putting $u_{i}:=2^{i} u_{0}$ for $1 \leq i \leq N$, we sum up over intervals $u_{i} \leq$ $x \leq u_{i+1}$. We have to choose $N$ such that $u_{N} \geq(2 T)^{1 / 2} \nu^{-1 / 2}$ or $N \geq$ $(4 \log 2)^{-1} \log T$. Since

$$
\frac{1}{a^{1 / 2} b^{3 / 2}} \cdot \frac{4 T}{u_{0}^{2} \nu}=\frac{4 T^{1 / 2}}{b(a+b)} \leq \frac{4 T^{1 / 2}}{b a}
$$


we find that the total number of suitable $(x, z)$ (and hence $(x, y))$ is

$$
\ll \frac{T^{1 / 2}}{a b}+\log T .
$$

Now, returning to the original problem and remembering that $\nu$ is a square $\mu^{2}$, we have to estimate

$$
\sum_{a b \leq T^{1 / 2}} \sum_{\mu^{2} \mid a^{2}-b^{2}}\left(\frac{T^{1 / 2}}{a b}+\log T\right) 2^{\omega\left(\left(a^{2}-b^{2}\right) / \mu^{2}\right)} .
$$

Fortunately, denoting by $d(n)$ the number of divisors of a positive integer $n$, we have

$$
\sum_{\mu^{2} \mid n} 2^{\omega\left(n / \mu^{2}\right)}=d(n)
$$

since both sides of the equation are multiplicative and the assertion is easily checked for powers of primes.

Let

$$
D_{b}(t)=\sum_{b<a \leq t} d\left(a^{2}-b^{2}\right),
$$

where it is understood that the sum runs over $a$ with $(a, b)=1$. This sum is

$$
\begin{aligned}
& \ll \sum d(a-b) d(a+b) \\
& \leq\left(\sum d(a-b)^{2}\right)^{1 / 2}\left(\sum d(a+b)^{2}\right)^{1 / 2} \leq \sum_{n \leq 2 t} d(n)^{2} .
\end{aligned}
$$

A well-known estimate shows (for $t \geq 2$, say) that this sum is $\ll t(\log t)^{3}$. So we have

$$
\begin{aligned}
\sum_{a b \leq T^{1 / 2}} d\left(a^{2}-b^{2}\right) & =\sum_{b \leq T^{1 / 4}} D_{b}\left(\frac{T^{1 / 2}}{b}\right) \\
& \ll T^{1 / 2}(\log T)^{3} \sum_{b \leq T^{1 / 4}} \frac{1}{b} \ll T^{1 / 2}(\log T)^{4} .
\end{aligned}
$$

On the other hand,

$$
\begin{aligned}
\sum_{a b \leq T^{1 / 2}} \frac{d\left(a^{2}-b^{2}\right)}{a b} & =\sum_{b \leq T^{1 / 4}} \frac{1}{b} \sum_{a \leq T^{1 / 2} b^{-1}} \frac{d\left(a^{2}-b^{2}\right)}{a} \\
& =\sum_{b \leq T^{1 / 4}} \frac{1}{b} \int_{b}^{T^{1 / 2} b^{-1}} \frac{1}{t} d D_{b}(t) .
\end{aligned}
$$

Integration by parts and trivial estimates show that the integral is « $(\log T)^{4}$, so the whole expression does not exceed $O\left((\log T)^{5}\right)$. Putting everything together, we have finished the proof of Proposition 2. 
We conclude by pointing out that this and the preceding proposition immediately imply

Corollary 3.

$$
\#\{n \leq T \mid a(n) \neq 0\}=C(4 T)^{3 / 4}+O\left(T^{1 / 2}(\log T)^{5}\right) .
$$

\section{References}

[1] D. Clark, An arithmetical function associated with the rank of elliptic curves, Canad. Math. Bull. 34 (1991), 181-185.

[2] J. Coates, Elliptic curves and Iwasawa theory, in: Modular Forms, R. Rankin (ed.), Halsted Press, New York, 1984.

[3] W. Jenkner, Asymptotic aspects of the Diophantine equation $p^{k} x^{n k}-z^{k}=l$, preprint.

[4] J. H. Silverman, The Arithmetic of Elliptic Curves, Springer, New York, 1986.

Institut für Mathematik

Universität Wien

Strudlhofgasse 4

1090 Wien, Austria

E-mail: wolfgang@mat.univie.ac.at 